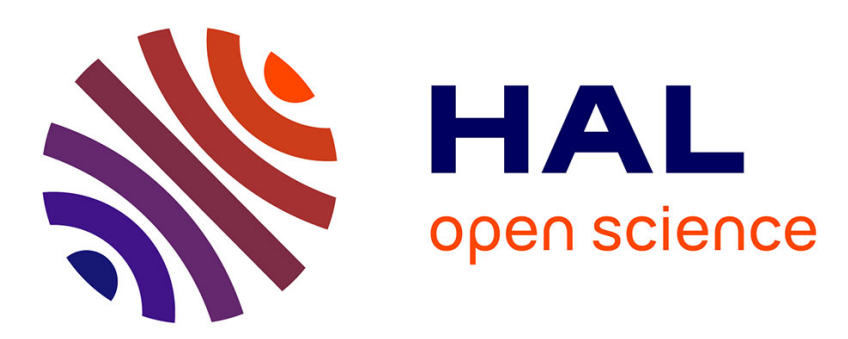

\title{
Hamiltonian tools for the analysis of optical polarization control
}

Elie Assemat, Antonio Picozzi, Hans-Rudolf Jauslin, Dominique Sugny

\section{To cite this version:}

Elie Assemat, Antonio Picozzi, Hans-Rudolf Jauslin, Dominique Sugny. Hamiltonian tools for the analysis of optical polarization control. Journal of the Optical Society of America B, 2012, 29 (4), pp.559-571. 10.1364/JOSAB.29.000559 . hal-00699914

\section{HAL Id: hal-00699914 \\ https://hal.science/hal-00699914}

Submitted on 21 May 2012

HAL is a multi-disciplinary open access archive for the deposit and dissemination of scientific research documents, whether they are published or not. The documents may come from teaching and research institutions in France or abroad, or from public or private research centers.
L'archive ouverte pluridisciplinaire $\mathbf{H A L}$, est destinée au dépôt et à la diffusion de documents scientifiques de niveau recherche, publiés ou non, émanant des établissements d'enseignement et de recherche français ou étrangers, des laboratoires publics ou privés. 


\title{
Hamiltonian tools for the analysis of optical polarization control
}

\author{
Elie Assémat, Antonio Picozzi, Hans-Rudolf Jauslin, and Dominique Sugny* \\ Laboratoire Interdisciplinaire Carnot de Bourgogne (ICB), UMR 5209 CNRS-Université de Bourgogne, \\ 9 Av. A. Savary, BP 47 870, F-21078 Dijon Cedex, France \\ ${ }^{*}$ Corresponding author: dominique.sugny@u-bourgogne.fr
}

Received October 18, 2011; revised December 2, 2011; accepted December 8, 2011; posted December 14, 2011 (Doc. ID 156777); published March 6, 2012

\begin{abstract}
The study of the polarization dynamics of two counterpropagating beams in optical fibers has recently been the subject of a growing renewed interest, from both the theoretical and experimental points of view. This system exhibits a phenomenon of polarization attraction, which can be used to achieve a complete polarization of an initially unpolarized signal beam, almost without any loss of energy. Along the same way, an arbitrary polarization state of the signal beam can be controlled and converted into any other desired state of polarization, by adjusting the polarization state of the counterpropagating pump beam. These properties have been demonstrated in various different types of optical fibers, i.e., isotropic fibers, spun fibers, and telecommunication optical fibers. This article is aimed at providing a rather complete understanding of this phenomenon of polarization attraction on the basis of new mathematical techniques recently developed for the study of Hamiltonian singularities. In particular, we show the essential role that play the peculiar topological properties of singular tori in the process of polarization attraction. We provide here a pedagogical introduction to this geometric approach of Hamiltonian singularities and give a unified description of the polarization attraction phenomenon in various types of optical fiber systems. (C) 2012 Optical Society of America
\end{abstract}

OCIS codes: $\quad 190.4370,060.4370,190.0190$.

\section{INTRODUCTION}

Achieving the repolarization of an optical field without loss is a fundamental physical phenomenon that can find a large variety of applications in telecommunication optical systems. In 2000, Heebner et al. proposed a "universal polarizer" performing polarization of unpolarized light with $100 \%$ efficiency, in contrast with conventional polarizers that unavoidably waste $50 \%$ of unpolarized light [1]. By using a photorefractive twobeam coupling, it was shown in Ref. [1] that unpolarized light can be converted to a polarized state with essentially a unit efficiency. This phenomenon has been termed "lossless polarization attraction," in the sense that all input polarization configurations are transformed into a particular polarization state, without any loss of energy. This kind of polarization attraction has been also identified in different nonlinear systems [2] and, in particular, in an optical fiber system pumped by two counterpropagating beams [3]. This latter phenomenon has been the subject of a growing interest these last years, from both the theoretical [ $\underline{4}-\underline{12}]$ and experimental [ $\underline{3}, \underline{13}-16]$ points of view. It finds its origin in pioneering studies of polarization dynamics of optical beams that counterpropagate in optical fibers and whose nonlinear interaction is mediated by the Kerr effect [17-27].

The recent works on polarization attraction in optical fibers reveal that the signal wave can be attracted toward a particular state of polarization (SOP) via a suitable choice of the injected SOP of the counterpropagating pump beam. An efficient polarization attraction has been shown to occur in different types of optical fibers, such as isotropic fibers (IFs) $[3,5,6,13]$, highly birefringent spun fibers (HBSF) [8,11], as well as randomly birefringent fibers (RBFs) used in optical telecommunication systems $[9,11,14]$. It is important to note that these phenomena of polarization attraction exhibit different properties that depend on the characteristics of the considered optical fiber. More precisely, when one considers IFs and RBFs, polarization attraction can take place either on a specific SOP, or on an ensemble of distinct SOPs $[6,11]$. On the other hand, in HBSFs, the attraction process has been recently shown to also occur on a specific line of polarization states that lie on the surface of the Poincaré sphere [11]. This remarkable and unexpected result is illustrated in Fig. 1. We considered here 64 different input states of polarization of the signal uniformly distributed over the Poincaré sphere (green points), while the SOP of the counterpropagating pump is kept fixed at the fiber output (yellow point). We see that all SOPs of the signal are attracted toward a specific line of polarization states at the fiber output (red points).

So far, most of the theoretical works aimed at describing this process of polarization attraction have focused on the derivation of the equations governing the evolution of the counterpropagating beams in different types of optical fibers, as well as on intensive numerical simulations of their spatiotemporal dynamics. However, little has been done in order to provide a theoretical description of the phenomenon of polarization attraction. Our aim in this article is to make a step in this direction by introducing a new set of tools that find their origin in recently developed mathematical techniques, in particular for the study of Hamiltonian singularities [28,29]. We have successfully used this mathematical approach in Refs. $[\underline{5}, \underline{6}]$ to study polarization attraction in IFs. We have recently extended this work to the polarization control in HBSFs and RBFs, the latter ones being used in optical 


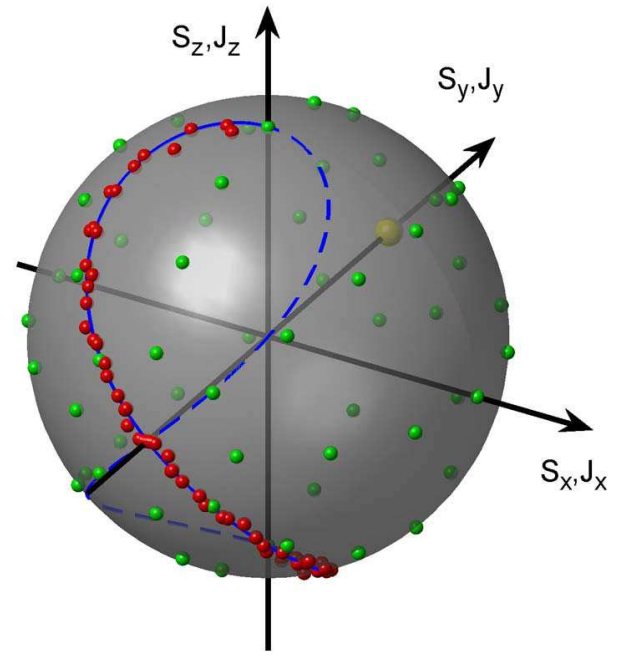

Fig. 1. (Color online) Polarization attraction toward a continuous line of polarization states in HSBFs. The attraction effect is demonstrated by integrating numerically the spatiotemporal Eq. (1) on the Poincaré sphere for $\varphi=\pi / 4$. The green and red dots denote respectively the initial $(\vec{S}(0))$ and final $(S(L))$ SOPs of the signal. The yellow dot represents the SOP of the pump, which is kept fixed at the fiber output: $\vec{J}(L)=(0,1,0)$ for a fiber length $L=5$. The blue line is calculated analytically in Sec. 4 . Note that the dashed part corresponds to unstable solutions that cannot be reached by the spatiotemporal dynamics.

telecommunication systems [11]. In this article we provide a unified geometrical description of these phenomena of polarization attraction. In particular, we show the essential role that the peculiar topological properties of singular tori play in the process of polarization attraction. This role is similar to the one played by the separatrix in purely one-dimensional systems [30]. We remark that the existence of these singular structures had been essentially ignored in the physics literature until their recent introduction in the domain of atomic and molecular systems $[\underline{29}, \underline{31}, \underline{32}]$. The aim of the present article is to render these new mathematical tools accessible to a broad audience in the context of nonlinear optics. In this respect, this paper can also be viewed as a pedagogical introduction to this geometric approach of Hamiltonian singularities, which may subsequently h2elp the interested reader to enter into a more specialized literature of Hamiltonian dynamics [28,29,33].

The paper is organized as follows. In Sec. 2 , we study the Hamiltonian structure of the stationary states of the spatiotemporal equations of wave propagation in nonlinear optics. In particular, we show that the corresponding Hamiltonian dynamics is integrable for the three different examples of optical fibers considered in this article. Section 3 is devoted to the derivation of the properties of the different singular tori, which allow a geometrical description of the stationary states. We show that the existence and the position of these singularities crucially depend on the characteristics of the optical fiber. In Sec. 4, we present the phenomenon of polarization attraction and the key role played by the singular tori. Some conclusions and discussions are presented in Sec. 5. Finally, considering the example of IFs, we show in the Appendix how a stationary soliton solution can be explicitly determined on the surface of a singular torus.

\section{INTEGRABLE HAMILTONIAN ON THE SPHERE}

The aim of this section is to introduce the specific Hamiltonian structure required to describe the stationary solutions of the phenomenon of polarization attraction in optical fibers. Before entering into the details, we underline that this mathematical approach is based on the following observation. Numerical simulations have revealed that, under rather general conditions, the spatiotemporal dynamics of the counterpropagating optical beams relaxes, after a complex transient, toward a stationary state. The theoretical approach discussed in this article provides a geometrical study of these stationary states. It reveals that they lie in the neighborhood of a singular torus of the corresponding phase-space representation. We shall see that it is the peculiar topological property inherent to the singular torus that enables a complete control of the polarization process.

The Hamiltonian formalism in a flat phase space (such as $\mathbb{R}^{2}$ ) is well known and has been widely employed in nonlinear optics in a large variety of systems. However, here the phasespace representation of the dynamics of polarization is the Poincaré sphere, which refers to the nonstandard phase space $S^{2} \times S^{2}$, i.e., a given point in this phase space corresponds to two vectors, $\vec{S}$ and $\vec{J}$ on the signal and pump Poincaré spheres. We have thus to adapt the conventional Hamiltonian formalism used in a flat phase space so as to use it with a spherical geometry.

The equations governing the polarization dynamics of the counterpropagating beams can be written in the following general form $[\underline{8}, \underline{9,}, \underline{27}]$ :

$$
\left\{\begin{array}{l}
\frac{\partial \vec{S}}{\partial t}+\frac{\partial \vec{S}}{\partial \xi}=\vec{S} \times\left(\mathcal{I}_{s} \vec{S}\right)+\vec{S} \times\left(\mathcal{I}_{i} \vec{J}\right) \\
\frac{\partial \vec{J}}{\partial t}-\frac{\partial \vec{J}}{\partial \xi}=\vec{J} \times\left(\mathcal{I}_{s} \vec{J}\right)+\vec{J} \times\left(\mathcal{I}_{i} \vec{S}\right)
\end{array},\right.
$$

where $\xi$ is the spatial coordinate along the fiber. The Stokes vectors $\vec{S}=\left(S_{x}, S_{y}, S_{z}\right)$ and $\vec{J}=\left(J_{x}, J_{y}, J_{z}\right)$ describe, respectively, the polarization states of the forward and backward beams on the Poincaré sphere and " $x$ " denotes the vector product. The matrices $\mathcal{I}_{s}$ and $\mathcal{I}_{i}$ are diagonal and their coefficients depend on the type of fibers considered [8,9]. The radii of the forward and backward spheres, $S_{0}$ and $J_{0}$, are the signal and pump powers, which are conserved quantities of the spatiotemporal dynamics. For convenience, we normalized the problem with respect to the characteristic nonlinear time $\tau_{0}=1 /\left(\gamma J_{0}\right)$ and length $\Lambda_{0}=v \tau_{0}$, where $\gamma$ is the nonlinear Kerr coefficient and $v$ the group-velocity of light in the optical fiber.

The introduction of the cylindrical coordinates

$$
\left\{\begin{array} { l } 
{ S _ { x } = \sqrt { S _ { 0 } ^ { 2 } - I _ { f } ^ { 2 } } \operatorname { c o s } \varphi _ { f } } \\
{ S _ { y } = \sqrt { S _ { 0 } ^ { 2 } - I _ { f } ^ { 2 } } \operatorname { s i n } \varphi _ { f } } \\
{ S _ { z } = I _ { f } }
\end{array} \quad \text { and } \quad \left\{\begin{array}{l}
J_{x}=\sqrt{J_{0}^{2}-I_{b}^{2}} \cos \varphi_{b} \\
J_{y}=\sqrt{J_{0}^{2}-I_{b}^{2}} \sin \varphi_{b} \\
J_{z}=-I_{b}
\end{array}\right.\right.
$$

allows us to define the standard Hamiltonian structure that makes use of the conventional Poisson brackets defined by

$$
\left\{g_{1}, g_{2}\right\}=\sum_{i=f, b} \frac{\partial g_{1}}{\partial \varphi^{i}} \frac{\partial g_{2}}{\partial I^{i}}-\frac{\partial g_{1}}{\partial I^{i}} \frac{\partial g_{2}}{\partial \varphi^{i}} .
$$


With this definition we have the following relations, $\left\{I_{b}, I_{f}\right\}=$ $\left\{\varphi_{b}, \varphi_{f}\right\}=0$ and $\left\{\varphi_{f}, I_{b}\right\}=\delta_{b f}$. In these coordinates the dynamics can be described by the usual Hamiltonian structure with a function $H\left(I_{b}, \varphi_{b}, I_{f}, \varphi_{f}\right)$ and the well-known Hamilton equations where $\xi$ plays here the role of time of the classical dynamical system:

$$
\begin{aligned}
& \partial_{\xi} I_{b, f}=\left\{I_{b, f}, H\right\}=-\frac{\partial H}{\partial \varphi_{b_{f} f}} \\
& \partial_{\xi} \varphi_{b_{f} f}=\left\{\varphi_{b, f}, H\right\}=\frac{\partial H}{\partial I_{b, f}} .
\end{aligned}
$$

However, these cylindrical coordinates are not globally defined, in the sense that when the polarization is circular, i.e., when $I_{f}^{2}$ (respectively $I_{b}^{2}$ ) is equal to $S_{0}^{2}$ (respectively $J_{0}^{2}$ ), then the angle $\varphi_{f}$ (respectively $\varphi_{b}$ ) is not defined. It turns out that the Stokes variables on the Poincare sphere are globally defined variables. The Poisson brackets can be expressed in the Stokes coordinates in the following form:

$$
\begin{aligned}
\left\{S_{x}, S_{y}\right\} & =\frac{\partial S_{x}}{\partial \varphi^{f}} \frac{\partial S_{y}}{\partial I^{f}}-\frac{\partial S_{x}}{\partial I^{f}} \frac{\partial S_{y}}{\partial \varphi^{f}} \\
& =S_{z} \\
& \ldots \\
\left\{J_{x}, J_{y}\right\} & =\frac{\partial J_{x}}{\partial \varphi^{b}} \frac{\partial J_{y}}{\partial I^{b}}-\frac{\partial J_{x}}{\partial I^{b}} \frac{\partial J_{y}}{\partial \varphi^{b}} \\
& =-J_{z},
\end{aligned}
$$

and we obtain by a circular permutation of the indices, the relations $\left\{S_{i}, S_{j}\right\}=\varepsilon_{i j k} S_{k}$ and $\left\{J_{i}, J_{j}\right\}=-\varepsilon_{i j k} J_{k}, \varepsilon_{i j k}$ being the completely antisymmetric tensor, i.e., it changes sign under the exchange of any pair of indices. Note that the minus sign in the $\vec{J}$ - equation is due to the counterpropagation of the two waves. These expressions of the Poisson brackets on the sphere in the Stokes coordinates are valid for any point of the sphere, which is not the case for the cylindrical coordinates.

The corresponding expression of the Hamiltonian also needs to be determined in the Stokes coordinates. We recall that, for a given Hamiltonian $H(\vec{S}, \vec{J})$, the dynamics of any functional (polynomial) $G(\vec{S}, \vec{J})$ is given by $\partial_{\xi} G=\{G, H\}$. Accordingly, the stationary system associated to Eq. (1) has the form

$$
\frac{d S_{i}}{d \xi}=\left\{S_{i}, H\right\} \quad \text { and } \quad \frac{d J_{i}}{d \xi}=\left\{J_{i}, H\right\}
$$

A constant of motion $K$ is independent of the time coordinate $\xi$ and is therefore characterized by the fact that it Poisson commutes with the Hamiltonian, i.e., $\{K, H\}=0$. From Eq. (3) and the above definition of the Poisson brackets on the sphere, it is straightforward to check that the Hamiltonian

$$
H=-\vec{S} \cdot \mathcal{I}_{i} \vec{J}-\frac{1}{2}\left(\vec{S} \cdot \mathcal{I}_{s} \vec{S}+\vec{J} \cdot \mathcal{I}_{s} \vec{J}\right)
$$

describes the stationary system of Eq. (1). We will now apply this formalism to three different models: the IF, the RBF, and the HBSF models.

Isotropic fiber. The diagonal matrices read $\mathcal{I}_{s}=$ $\operatorname{diag}(-1,-1,0)$ and $\mathcal{I}_{i}=\operatorname{diag}(-2,-2,0)$. The stationary system is given by

$$
\left\{\begin{array}{l}
\partial_{\xi} S_{x}=S_{z} S_{y}+2 S_{z} J_{y} \\
\partial_{\xi} S_{y}=-S_{z} S_{x}-2 S_{z} J_{x} \\
\partial_{\xi} S_{z}=2 J_{x} S_{y}-2 S_{x} J_{y}
\end{array}\right.
$$

and

$$
\left\{\begin{array}{l}
\partial_{\xi} J_{x}=-J_{z} J_{y}-2 J_{z} S_{y} \\
\partial_{\xi} J_{y}=J_{z} J_{x}+2 J_{z} S_{x} \\
\partial_{\xi} J_{z}=2 J_{x} S_{y}-2 S_{x} J_{y}
\end{array} .\right.
$$

Up to an additive constant, the Hamiltonian thus takes the form

$$
H=2\left(S_{y} J_{y}+S_{x} J_{x}\right)-\frac{1}{2}\left(S_{z}^{2}+J_{z}^{2}\right)
$$

and we also remark that $K=S_{z}-J_{z}$ is a constant of motion since $\{H, K\}=0$.

Randomly birefringent fiber. The diagonal matrices read $\mathcal{I}_{s}=0$ and $\mathcal{I}_{i}=\operatorname{diag}(-1,1,-1)$ [9]. The stationary system can be written as

$$
\left\{\begin{array}{l}
\partial_{\xi} S_{x}=-S_{y} J_{z}-S_{z} J_{y} \\
\partial_{\xi} S_{y}=-J_{x} S_{z}+S_{x} J_{z} \\
\partial_{\xi} S_{z}=J_{y} S_{x}+S_{y} J_{x}
\end{array}\right.
$$

and

$$
\left\{\begin{array}{l}
\partial_{\xi} J_{x}=S_{y} J_{z}+J_{y} S_{z} \\
\partial_{\xi} J_{y}=J_{z} S_{x}-S_{z} J_{x} \\
\partial_{\xi} J_{z}=-J_{y} S_{x}-J_{x} S_{y}
\end{array} .\right.
$$

The Hamiltonian becomes

$$
H=S_{x} J_{x}-S_{y} J_{y}+S_{z} J_{z}
$$

and it Poisson commutes with the three constants of motion $K_{1}=S_{x}+J_{x}, K_{2}=S_{y}-J_{y}$, and $K_{3}=S_{z}+J_{z}$.

Highly birefringent spun fiber. The diagonal matrices read $\mathcal{I}_{s}=\operatorname{diag}(0,0, \beta)$ and $\mathcal{I}_{i}=\alpha \operatorname{diag}(1,-1,-2)$, where $\alpha=\cos ^{2} \varphi$, $\beta=2 \sin ^{2} \varphi-\cos ^{2} \varphi$, and $\varphi$ is the ellipticity of the fiber [8]. The stationary system reads

$$
\left\{\begin{array}{l}
\partial_{\xi} S_{x}=\alpha\left(S_{z} J_{y}-2 S_{y} J_{z}\right)+\beta S_{y} S_{z} \\
\partial_{\xi} S_{y}=\alpha\left(S_{z} J_{x}+2 S_{x} J_{z}\right)-\beta S_{x} S_{z} \\
\partial_{\xi} S_{z}=-\alpha\left(J_{y} S_{x}+S_{y} J_{x}\right)
\end{array}\right.
$$

and

$$
\left\{\begin{array}{l}
\partial_{\xi} J_{x}=\alpha\left(2 S_{z} J_{y}-S_{y} J_{z}\right)-\beta J_{y} J_{z} \\
\partial_{\xi} J_{y}=-\alpha\left(2 S_{z} J_{x}+S_{x} J_{z}\right)+\beta J_{x} J_{z} \\
\partial_{\xi} J_{z}=\alpha\left(J_{y} S_{x}+J_{x} S_{y}\right),
\end{array}\right.
$$

leading to the Hamiltonian

$$
H=\alpha\left(S_{y} J_{y}-S_{x} J_{x}+2 S_{z} J_{z}\right)-\frac{\beta}{2}\left(S_{z}^{2}+J_{z}^{2}\right)
$$

that Poisson commutes with $K=S_{z}+J_{z}$.

These three models are Liouville-integrable [34], i.e., they possess at least as many invariants as the dimension of the phase space divided by two. In our study the phase space 
is $S^{2} \times S^{2}$, which is described locally by $\left(I_{f}, \varphi_{f}, I_{b}, \varphi_{b}\right)$, and each model discussed here above has at least two invariants, $H$ and $K$. One of the important consequences of the integrability is the fact that the dynamics curls around a torus in the phase space. This means that there exists a set of action-angle coordinates $(J, \psi, K, \chi)$ such that the two actions are constant on the corresponding torus and the dynamics is described only by the two angles $(\psi, \chi)$. In these coordinates, the two actions are associated to the two radii of a torus and the two angles to the angles needed to describe a trajectory on the surface of the torus. This torus can be either regular or singular, as illustrated in Fig. 2. The regular torus generically leads to an oscillating behavior of the dynamics, while the singular torus describes more specific trajectories that exhibit a "monotonous" behavior. In this respect a singular torus can be regarded as a two-dimensional generalization of the well-known separatrix in purely one-dimensional systems. The singular tori play a key role in the phenomenon of polarization attraction and the next section is devoted to their characterization.

\section{CONSTRUCTION OF THE SINGULAR TORI}

In order to study the Hamiltonian systems established in the previous section, we introduce here the singular reduction technique. Its objective is to reduce the number of dimensions of the system by using the constant of motion $K$, which gives information on the nature of the singularities of the system. As we will see, this analysis provides all the material required to understand the polarization attraction phenomenon in the different types of optical fiber systems. A simple introduction to the reduction theory is presented below. We refer the reader to more specialized mathematical books for a more rigorous mathematical presentation of the theory [28].

(a)

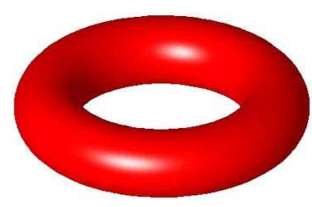

(b)

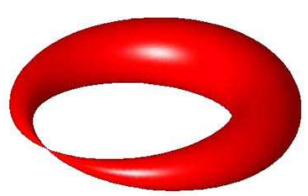

(c)

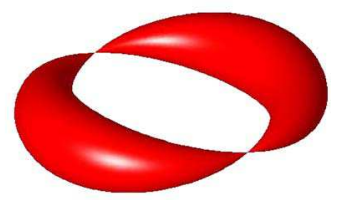

(d)

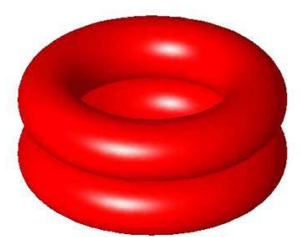

(e)

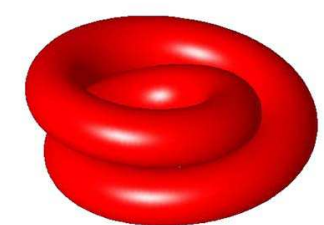

Fig. 2. (Color online) Examples of tori of an integrable Hamiltonian phase space of dimension four. A system can freely oscillate around a regular (standard) torus (a), but its evolution can also be blocked by the presence of a pinched point in a singly (b) or doubly (c) pinched torus. The singular pinched tori can be viewed as a two-dimensional generalization of the concept of separatrix, well-known for systems with 1 degree of freedom. A bitorus and a curled torus are represented in (d) and (e). They can be constructed by gluing two regular tori along a circle, with an additional twist in the case of a curled torus.
In simple terms, the reduction theory can be viewed as a change of variables that exploits the constant of the motion $K$ to reduce the dimensionality of the problem. The lower dimensional phase space obtained by the reduction theory will be called reduced phase space. We reported in Fig. 3 a schematic representation of the original phase space $S^{2} \times \bar{S}^{2}$ and of the reduced phase space. The new variables of the reduced phase space need to be defined globally and in a way as simple as possible. This explains why we first reduce the dimension of the system with respect to the constant $K$, which has a simpler form than $H$. The new coordinates have, by definition, a dynamic that belongs to a given $K$-invariant subspace, which means that they should Poisson commute with $K$. These coordinates called invariant polynomials are denoted by $\left(x_{0}, x_{1}, x_{2}, x_{3}\right)$. Note that, in general, such coordinates are not constants for the $H$ dynamics, but only for the $K$ dynamics defined by

$$
\frac{d S_{i}}{d z}=\left\{S_{i}, K\right\} \quad \text { and } \quad \frac{d J_{i}}{d z}=\left\{J_{i}, K\right\}
$$

which is just another way to express the fact that they Poisson commute with $K$. The dynamical system defined by Eq. (ㅁ) is different from the physical system of interest. We may just consider it as a procedure to visualize the influence of the constant of motion $K$ on the dynamics.

Isotropic fiber. Let us begin to illustrate the reduction technique with the example of the IF system. Equation ( $\underline{8}$ ) and the constant of motion $K=S_{z}-J_{z}$ lead to the differential system

$$
\left\{\begin{array} { l } 
{ \dot { S } _ { y } = S _ { x } } \\
{ \dot { S } _ { x } = - S _ { y } ; } \\
{ \dot { S } _ { z } = 0 }
\end{array} \quad \left\{\begin{array}{l}
\dot{J}_{y}=J_{x} \\
\dot{J}_{x}=-J_{y} \\
\dot{J}_{z}=0
\end{array}\right.\right.
$$

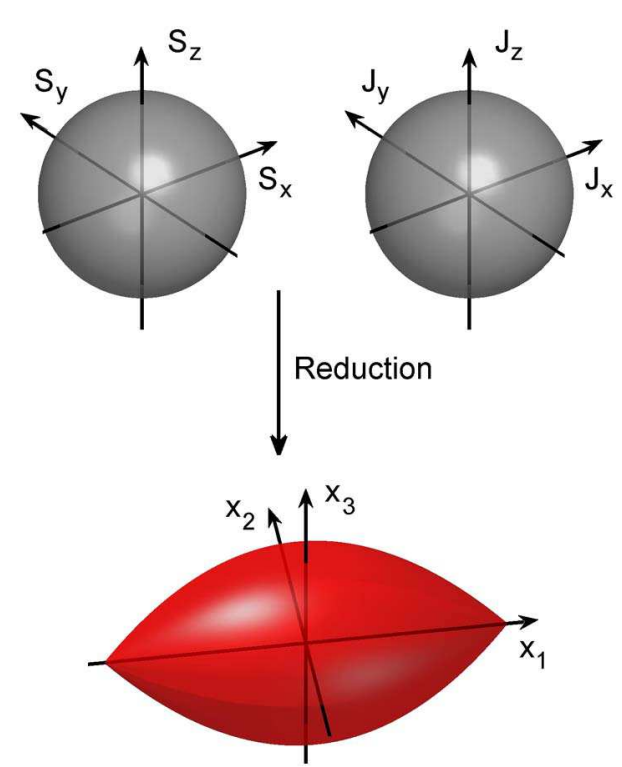

Fig. 3. (Color online) Schematic illustration of the reduction process, which maps the main phase space, i.e., the two Poincaré spheres, $S^{2} \times S^{2}$, toward the reduced phase space, which has the form of a deformed sphere with two conical singularities. The reduced phase space is defined by Eq. (10) for the IF and Eq. (13) for the RBF and HBSF. 
These equations simply describe circles in the main phase space: they correspond to a simultaneous rotation of $\vec{S}$ and $\vec{J}$ around the $z$ axis with the same angular velocity. The idea of the reduction theory is to get rid of this trivial circular dynamic by projecting it to a point in the reduced phase space, as illustrated in Fig. $\underline{3}$ and Fig. $\underline{5}$. In order to determine the coordinates of the reduced phase space, we have to consider variables that are invariant under Eq. (9), i.e., such that they Poisson commute with $K$. It can be shown that the reduced phase space can be expressed in terms of four coordinates $\left(x_{0}, x_{1}, x_{2}, x_{3}\right)$ with a relation between them, which leads, as expected, to a three-dimensional space. $K$ being the Hamiltonian of the system (9), it can be chosen as one of the invariant polynomials, $x_{0}$. The other three can be found by analyzing Eq. (9). It is straightforward to check that a possible set of invariant polynomials is

$$
\left\{\begin{array}{l}
x_{0}=K=S_{z}-J_{z} \\
x_{1}=S_{z}+J_{z} \\
x_{2}=\vec{S} . \vec{J} \\
x_{3}=S_{x} J_{y}-S_{y} J_{x}
\end{array} .\right.
$$

The new coordinates obey the relation

$$
\begin{aligned}
x_{3}^{2} & +\left(x_{2}+\frac{1}{4}\left(x_{0}^{2}-x_{1}^{2}\right)^{2}\right)^{2} \\
& -\left(S_{0}^{2}-\frac{1}{4}\left(x_{0}+x_{1}\right)^{2}\right)\left(J_{0}^{2}-\frac{1}{4}\left(x_{0}-x_{1}\right)^{2}\right)=0,
\end{aligned}
$$

with the constraint $-S_{0}-J_{0} \leq x_{1} \leq S_{0}+J_{0}$, which simply originates in the definitions of the Stokes coordinates. It can be shown that the variables $\left(x_{0}, x_{1}, x_{2}, x_{3}\right)$ constitute a basis for the set of polynomial functions commuting with $K$ [29]. In particular, since $\{H, K\}=0$, one can write the Hamiltonian as

$$
H=2 x_{2}+\frac{1}{4} x_{0}^{2}-\frac{3}{4} x_{1}^{2}
$$

For each fixed $K$, Eq. (10) defines a surface in the space $\left(x_{1}, x_{2}, x_{3}\right)$, which is called the reduced phase space. It is depicted in red in Figs. $\underline{4}$ and $\underline{5}$. For each value of $(H, K)$, Eq. (11) defines a second surface, called Hamiltonian surface, and is represented in blue in Fig. 4. The intersection of these two surfaces gives the trajectories of the system. To recover the dynamics in the main phase space from the dynamics in the reduced phase space, we have to make the Cartesian product of the reduced dynamics by the circle that comes from Eq. (9). For example, if the dynamics follow a circle in the reduced phase space, a Cartesian product with a second circle leads to a regular torus [see Fig. 2(a)]. The fact that each point of the reduced phase space corresponds to a circle in the main phase space holds true as long as the derivative on the reduced phase space is continuous, i.e., as long as the surface is smooth. This is illustrated in Fig. 4. The surface is plotted with $K=0$ and $S_{0}=J_{0}$, thus $S_{z}=\bar{J}_{z}$. The two nonsmooth points are of coordinates $\left(x_{1}= \pm 2, x_{2}=1, x_{3}=0\right)$. This entails $S_{z}=J_{z}=S_{0}$ or $S_{z}=J_{z}=-S_{0}$. If we report this result in Eq. (9), we see that the motion is no longer circular but stationary, i.e., it refers to a point. This clearly illustrates the following important aspect: when a point of the reduced



Fig. 4. (Color online) IF model: Intersection of the reduced phase space (red) and of the Hamiltonian surface (blue) with $S_{0}=J_{0}=1, K=0$, and $H=-1$. We observe that the reduced phase space for $K=0$ has two points with noncontinuous derivative. The intersection with the Hamiltonian surface $H=$ cst contains both points, which as a consequence is a singular torus (see the text).

phase space is not smooth, then this point does not correspond to a circle in the main phase space $S^{2} \times S^{2}$, but to a stationary point.

Let us illustrate this fact with the concrete example of the IF, in which, for simplicity, we consider the case $S_{0}=J_{0}=1$. The surfaces of Eq. (10) and Eq. (11) are represented in Fig. 4 for $(H=-1, K=0)$. The intersection contains two nonsmooth points $\left(x_{1}= \pm 2, x_{2}=1, x_{3}=0\right)$; thus, the

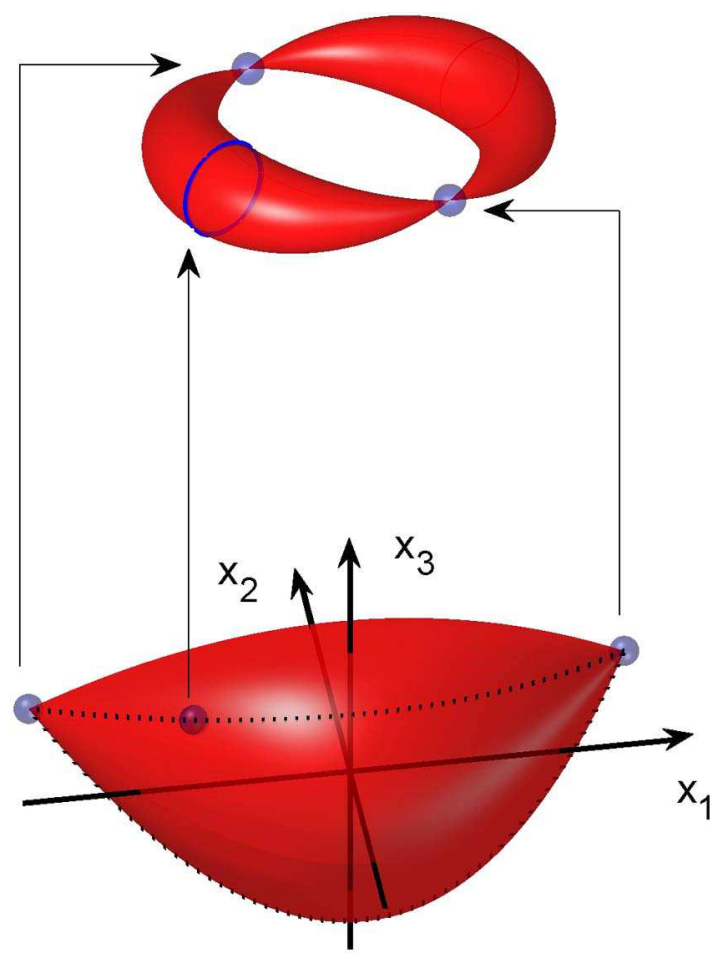

Fig. 5. (Color online) IF model: Illustration of the relation between the points of the reduced phase space and the points of the singular torus. The dashed line depicts the intersection with the Hamiltonian surface. 
corresponding set in the original phase space is a singular doubly pinched torus (see Fig. 5). One can plot these two surfaces for different values of $(H, K)$, and verify that there is no other isolated singularity. These results are summarized in the so-called energy-momentum diagram of Fig. $\underline{6}$. Such a diagram is the ensemble of all the possible values of $H$ and $K$ for some given fixed values of the radii $S_{0}$ and $J_{0}$ of the Stokes spheres. It provides a global view of the stationary states of the system. Following the previous geometric analysis, and according to the Liouville-Arnold theorem, any point of this set will be associated either to a regular or to a singular torus.

The equation of the boundary of this diagram can be obtained from the property that, at the boundary, the intersection of the reduced phase space and of the Hamiltonian surface reduces to a point. The computation goes as follows. First, one notices that the symmetry of the two surfaces implies that such an intersection occurs when $x_{3}=x_{1}=0$. We then express from Eq. (10) the polynomial $x_{3}^{2}\left(x_{1}\right)$ and we compute its roots:

$$
x_{1}= \pm \sqrt{\frac{2 S_{0}^{4}-S_{0}^{2} K^{2}-K^{2} x_{2}-2 x_{2}^{2}}{S_{0}^{2}-x_{2}}} .
$$

Finally, using Eq. (11) and the fact that $x_{1}=0$ we get

$$
H=2 S_{0}^{2}-\frac{3}{4} K^{2} \quad \text { and } \quad H=-2 S_{0}^{2}+\frac{1}{4} K^{2} .
$$

Note that for the IF model this diagram presents only one isolated singularity. This interesting property has a direct influence of the polarization attraction phenomenon, as explained in the next section.

Randomly birefringent fiber. We now analyze the example of RBFs by following the same procedure as that outlined here above for IFs. In the case of RBFs, we need to choose a constant of motion among the three available ones in order to carry out the reduction process. Note that this arbitrary choice has no consequence on the properties of the energymomentum diagram. The choice $K_{3}=S_{z}+J_{z}$ and Eq. ()), leads to

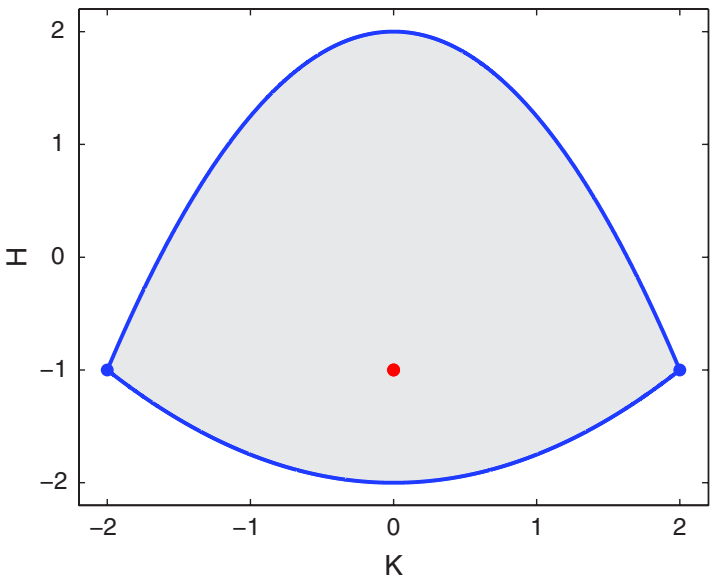

Fig. 6. (Color online) IF model: Energy-momentum diagram for $S_{0}=J_{0}=1$. The gray region corresponds to regular tori in the phase space and the red dot to a doubly pinched torus.

$$
\left\{\begin{array}{l}
\dot{S}_{x}=-S_{y} \\
\dot{S}_{y}=S_{x} \\
\dot{S}_{z}=0
\end{array} ; \quad\left\{\begin{array}{l}
\dot{J}_{x}=J_{y} \\
\dot{J}_{y}=-J_{x}, \\
\dot{J}_{z}=0
\end{array}\right.\right.
$$

and the following polynomials are constant under this dynamic:

$$
\left\{\begin{array}{l}
x_{0}=K_{3}=S_{z}+J_{z} \\
x_{1}=S_{z}-J_{z} \\
x_{2}=S_{y} J_{y}-S_{x} J_{x} \\
x_{3}=S_{x} J_{y}+S_{y} J_{x}
\end{array}\right.
$$

These invariant polynomials fulfill the relation:

$$
x_{3}^{2}+x_{2}^{2}+\left(S_{0}^{2}-\frac{1}{4}\left(x_{0}+x_{1}\right)^{2}\right)\left(J_{0}^{2}-\frac{1}{4}\left(x_{0}-x_{1}\right)^{2}\right)=0 .
$$

The Hamiltonian can be rewritten in the form:

$$
H=\frac{1}{4}\left(x_{0}^{2}-x_{1}^{2}\right)-x_{2} .
$$

Here again, we consider $S_{0}=J_{0}$, which leads to a unique value of $\left(H, K_{3}\right)=\left(-S_{0}^{2}, 0\right)$ for which the intersection is not smooth. Furthermore, if we report this value in Eqs. (13) and (14), both give the relation $x_{2}=S_{0}^{2}-x_{1}^{2} / 4$, i.e., the intersection is a segment, as can be seen in Fig. 8 . The two edges of the segment are nonsmooth points of the reduced phase space and correspond therefore to points in the main phase space, while the others are regular and are thus associated to circles in the main phase space. By collecting the different results, we finally obtain a singular torus, which is topologically equivalent to a sphere. Indeed, the two edges of the intersection produce the two poles of the sphere and the other points of the segment correspond to the different parallel circles of the sphere (see Fig. 8). This sphere plays the same role as the singular torus for the IF case.

Let us now construct the energy-momentum diagram by using the same symmetry as for the case of IFs, $x_{3}=x_{1}=0$. Straightforward computations lead to the diagram of Fig. 7, whose boundary is determined by the equation

$$
H=\frac{K_{3}^{2}}{2}-S_{0}^{2} \quad \text { and } \quad H=S_{0}^{2}
$$

A distinguished feature of this diagram is the fact that the singular torus is on the border of the domain. This property will be shown to influence the phenomenon of polarization attraction.

Highly birefringent spun fiber. In this case, the Hamiltonian depends on a real parameter $\varphi$, which gives rise to a family of reduction procedures. The constant of motion $K=$ $S_{z}+J_{z}$ is the same as that used for RBFs. Accordingly, one can use the same invariant polynomials for this system, which thus leads to the same reduced phase space. The Hamiltonian now reads

$$
H=\alpha\left(x_{2}+\frac{x_{0}^{2}-x_{1}^{2}}{2}\right)-\frac{\beta}{4}\left(x_{0}^{2}+x_{1}^{2}\right) .
$$




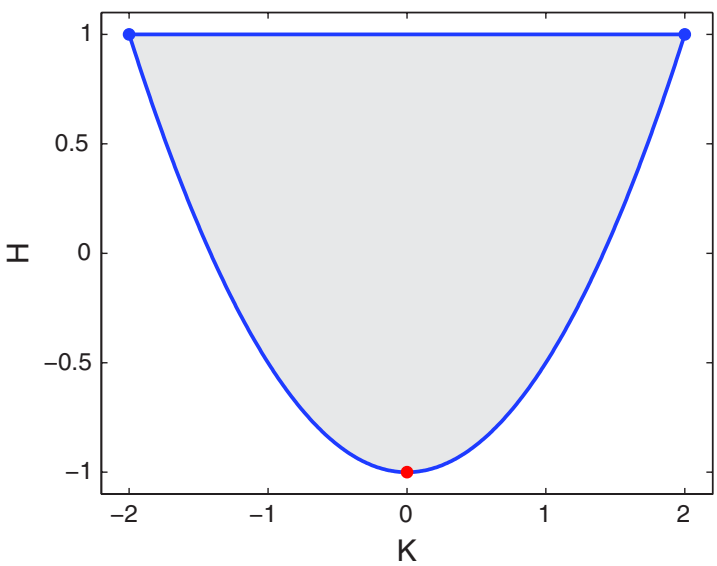

Fig. 7. (Color online) RBF model: Energy-momentum diagram for $S_{0}=J_{0}=1$. The gray region corresponds to regular tori in the phase space and the red dot to a sphere.

Let us consider again the case $S_{0}=J_{0}$. The intersection of the reduced phase space and the Hamiltonian surface is reported in Fig. 9. It exhibits an eight-shaped intersection, which corresponds in the main phase space to a bitorus [see Fig. 2(d)], which is the union of two tori glued along a circle. Indeed, if we rotate this eight-shaped intersection around the circle of Eq. (12) in a three-dimensional space, we obtain a bitorus. The upper torus of the bitorus is drawn by the upper loop of the eight, and the same goes for the lower part of the eight.

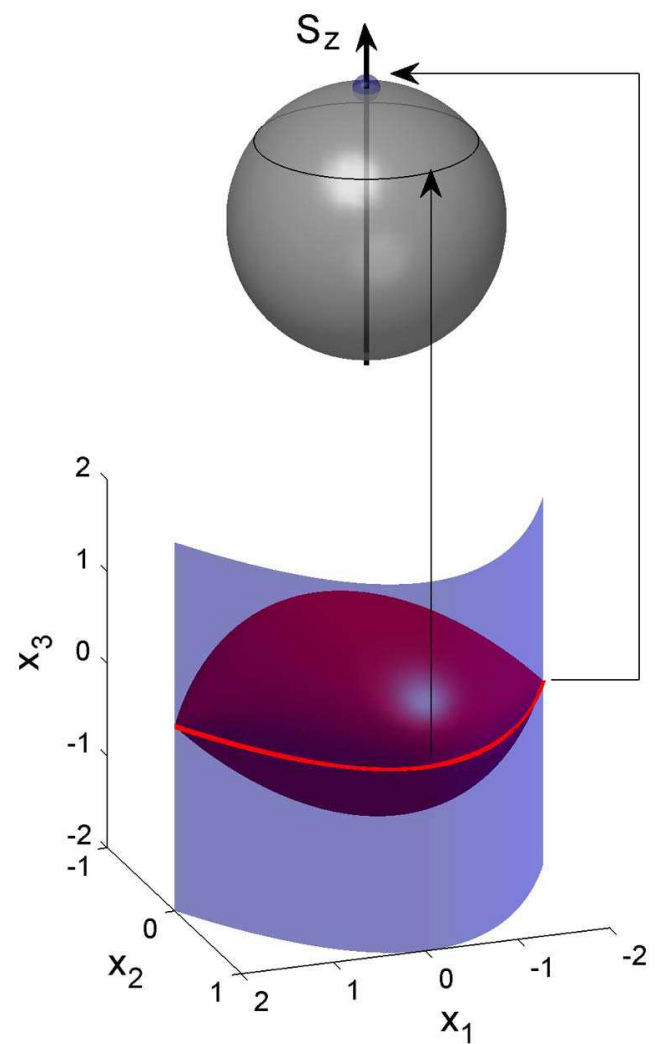

Fig. 8. (Color online) RBF model: The Hamiltonian surface (blue surface) and the reduced phase space (red surface) intersect along a segment (red line) that draws a sphere in the main phase space.

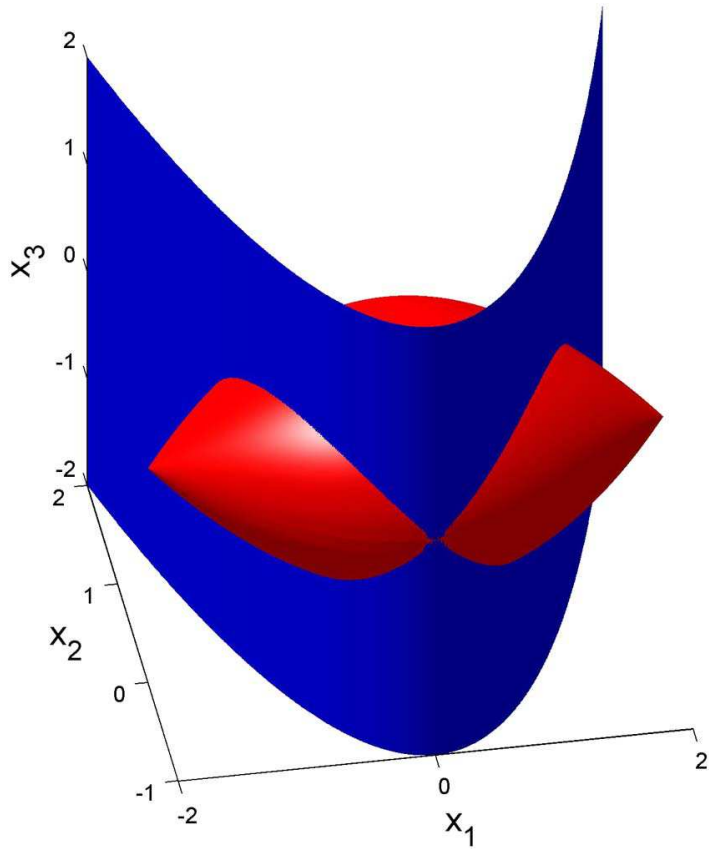

Fig. 9. (Color online) HBSF model: Intersection of the reduced phase space defined by Eq. (13) and the Hamiltonian of Eq. (15) with $\varphi=\pi / 4, K=0$, and $H=-\overline{0.5}$.

We now discuss the energy-momentum diagram. In contrast with the examples of the IF and the RBF, whose energy-momentum diagrams were characterized by an isolated singularity, here the diagram is characterized by a continuous line of singular bitori. In other terms, we have an infinite number of values of $(H, K)$ that produce a bitorus. These values draw a line in the $(H, K)$ plane and it is possible to derive the equation of this line explicitly. Here again the symmetries of the problem lead to $x_{3}=x_{1}=0$. We first compute the roots of the polynomial $x_{3}^{2}\left(x_{1}\right)$ :

$$
x_{1}= \pm \sqrt{K^{2}+4 S_{0}^{2} \pm 4 \sqrt{S_{0}^{2} K^{2}+x_{2}^{2}}}
$$

Then we use $x_{1}=0$ and Eq. ( $\left.\underline{15}\right)$ to eliminate $x_{2}$ and after a few steps of simple calculations we obtain

$$
\left\{\begin{array}{l}
H=\varepsilon \sqrt{\left.\alpha^{2}\left(\frac{K^{2}}{4}+S_{0}^{2}\right)^{2}-S_{0}^{2} K^{2}\right)}+\frac{\alpha K^{2}}{2}-\frac{\beta K^{2}}{4} \\
H=-2 \alpha S_{0}^{2}-S_{0}^{2} \beta-\frac{\beta K^{2}}{2}+|K| \sqrt{4 \alpha \beta S_{0}^{2}+S_{0}^{2} \beta^{2}+3 S_{0}^{2} \alpha^{2}}
\end{array},\right.
$$

where $\varepsilon= \pm 1$. The first equation of (16) provides the red line $(\varepsilon=-1)$ and the upper $(\varepsilon=+1)$ blue line of Fig. 10, while the second relation provides the lower blue lines. The main line of singularities in the energy-momentum (red line in Fig. 10) diagram can be simplified into

$$
H=\cos ^{2} \varphi\left(K^{2}-S_{0}^{2}\right)-\frac{\sin ^{2} \varphi}{2} K^{2} .
$$






Fig. 10. (Color online) HBSF model: Energy-momentum diagram with $\varphi=\pi / 4$. Each point of the gray region corresponds to a regular torus in the main phase space and each point of the red line corresponds to a bitorus.

\section{POLARIZATION ATTRACTION}

In this section we study the polarization attraction phenomenon in the light of the singular reduction theory exposed above for the three examples of fiber systems.

Isotropic fiber. This case has been the subject of a detailed study in [6] and [10], respectively, for the phenomenon of polarization attraction and the issue of the soliton stability. We thus refer the reader to these two articles for more details. Here, we illustrate how the previously introduced mathematical tools can be applied to analyze the process of polarization attraction. First, we consider the following change of variables :

$$
\left\{\begin{array}{l}
S_{x}=\sqrt{S_{0}^{2}-I_{f}^{2}} \cos \varphi_{f} \\
S_{y}=\sqrt{S_{0}^{2}-I_{f}^{2}} \sin \varphi_{f} \\
S_{z}=I_{f}
\end{array} ; \quad\left\{\begin{array}{l}
J_{x}=\sqrt{J_{0}^{2}-I_{b}^{2}} \cos \varphi_{b} \\
J_{y}=\sqrt{J_{0}^{2}-I_{b}^{2}} \sin \varphi_{b} \\
J_{z}=-I_{b}
\end{array}\right.\right.
$$

to rewrite the Hamiltonian in the following form

$$
H=2 \sqrt{\left(S_{0}^{2}-I_{f}^{2}\right)\left(S_{0}^{2}-I_{b}^{2}\right)} \cos \left(\varphi_{f}-\varphi_{b}\right)-\frac{I_{f}^{2}+I_{b}^{2}}{2} .
$$

Next, we denote by $J_{z}(z=L)=e$ the ellipticity of the pump wave injected into the fiber at $z=L$. We assume that the spatiotemporal system relaxes toward the only singularity of the energy-momentum diagram (see Fig. 6 ), thus $K=S_{z}-J_{z}=0$ and $H=-S_{0}^{2}$. This implies that the signal is attracted toward the same ellipticity as the pump wave $S_{z}(L)=e$. Finally, we use $H=-S_{0}^{2}$ to calculate the orientation of the polarization ellipse to which the signal is attracted, i.e., the angle $\varphi_{f}(L)$ in the plane $S_{z}=e$ :

$$
\left(S_{0}^{2}-e^{2}\right)\left(2 \cos \left(\varphi_{f}-\varphi_{b}\right)+1\right)=0 .
$$

We have to consider two different cases. If the polarization of the pump is circular $\left(e=J_{0}\right)$, then the signal is attracted toward the same circular polarization state. On the other hand, if the polarization of the pump is elliptic, then the orientation of the polarization ellipse of the signal is related to that of the pump by

$$
\varphi_{f}=\varphi_{b} \pm \frac{2 \pi}{3}
$$

This result was confirmed by the numerical simulations of the spatiotemporal Eqs. (1) in Ref. [6].

Randomly birefringent fiber. The application of the preceding mathematical tools to the polarization attraction in the RBFs was briefly discussed in Ref. [11]. Here, we summarize the main results obtained in [11] and also discuss the influence of the powers of the beams on the robustness of the attraction process (note that, with the adopted normalization it is equivalent to increase the beam power or the fiber length).

We first consider the case $S_{0}=J_{0}$. Using the reduction theory of Sec. 3 , we know by symmetry of the directions that, on the singular torus, we have $K_{1}=K_{2}=K_{3}=0$. It is then straightforward to deduce the polarization attraction relations: $S_{1}(L)=-J_{1}(L), S_{2}(L)=J_{2}(L)$, and $S_{3}(L)=-J_{3}(L)$. This theoretical prediction was also confirmed by the numerical simulations of the spatiotemporal Eqs. (1) in Ref. [11]. Note that the main difference between the RBF and IF is the fact that the singular torus lies on the boundary of the energy-momentum diagram (see Fig. 7). This strongly influences the efficiency of the attraction process, as illustrated by the comparison of the distance of the stationary solution to the singular torus for IFs and RBFs. For example, the average distance $\rho=\sqrt{(H+1)^{2}+K^{2}}$ is $10^{-2}$ in the RBF (with $L=15$ ), whereas it is $10^{-7}$ in the IF case (with $L=5$ ). Furthermore, when one considers IFs, the distance $\rho$ decreases exponentially with the fiber length $L$ [5]. Conversely, for RBFs, the distance $\rho$ also decreases as $L$ increases, but in a slower way. This is illustrated in Fig. 11. It can be interpreted by considering the fact that the singular torus lies on the boundary of the energy-momentum diagram for RBFs, while it is located inside the diagram for IFs. Then contrary to IFs, convergence toward the singular torus cannot occur in an isotropic way in RBFs, which limits the accuracy of the polarization attraction phenomenon.

Finally note that a power difference $\left(S_{0} \neq J_{0}\right)$ can strongly affect the attraction process. We performed numerical simulations with different values of $\Delta=\left(S_{0}-J_{0}\right) / S_{0}$. The numerical study reveals that for $\Delta$ in the range of few percents, the system still relaxes toward a stationary state, as illustrated in Fig. 12. For larger values of $\Delta$, the spatiotemporal dynamics no longer converge toward a stationary state. Note that there is not a clear threshold on $\Delta$. When $\Delta$ increases we

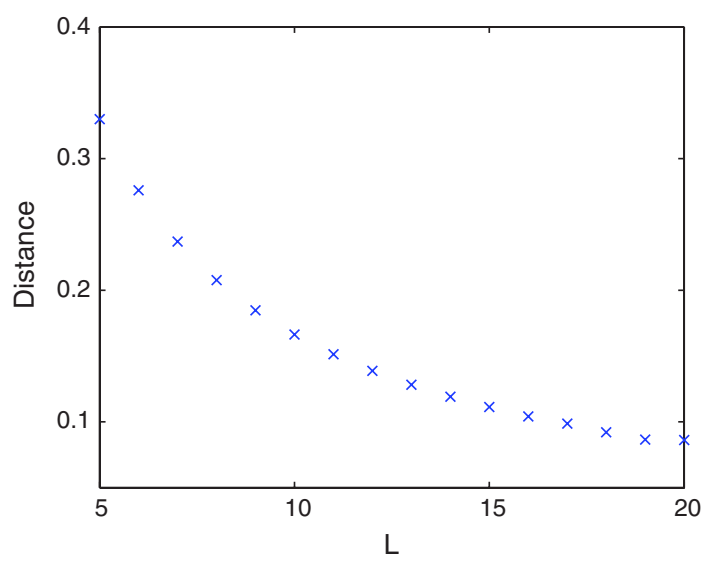

Fig. 11. (Color online) RBF model: The distance of the pump SOP to the predicted point decreases as $L$ increases. The distance plotted here is the average of the distances of the 64 different signal inputs uniformly distributed on the Poincaré sphere (green dots in Fig. 1) with $J_{x}(L)=J_{y}(L)=0.7$ and $J_{z}(L)=\sqrt{2} / 10$. 
numerically observe that the solution with $\vec{S}(t=0, z=0)$ close to $\vec{J}(t=0, z=0)$ cannot relax toward a stationary state while all the others do. When $\Delta$ is large enough there is no stationary state, for any initial condition.

Highly birefringent spun fiber. The application of the theoretical tools exposed in the preceding section to the process of polarization attraction in HBSFs was briefly discussed in Ref. [11]. Here, we complete our results and also suggest a possible application of this system.

From the theoretical point of view, the case of HBSFs is fundamentally different from IFs or RBFs. Indeed, as explained in Sec. 3, instead of an isolated singularity, the energy-momentum diagram of HSBF exhibits a continuous line of singularities. The direct consequence of this property is the fact that the signal beam is no longer attracted toward a single SOP, but instead toward a line of polarization states that lie on the surface of the Poincaré sphere.

Let us illustrate this result by considering the particular values $\varphi=\pi / 4$ and $\left[J_{x}(z=L)=0, J_{y}(z=L)=1, J_{x}(z=\right.$ $L)=0$ ]. Indeed, in this particular case, one can obtain a simple analytical expression of the line of singularities on the Poincaré sphere. For this purpose, we substitute in Eq. (7) the expression $S_{x}=\sqrt{S_{0}^{2}-S_{y}^{2}-S_{z}^{2}}$ and $S_{z}=K-J_{z}$ to get

$$
S_{y}=K^{2}-S_{0}^{2} \text {. }
$$

Then using $J_{z}=0$ and $S_{x}=\sqrt{S_{0}^{2}-S_{y}^{2}-S_{z}^{2}}$, we get

$$
S_{x}= \pm \sqrt{S_{0}^{2}-K^{2}-\left(S_{0}^{2}-K^{2}\right)^{2}} .
$$

Since $J_{z}=0$ gives $S_{z}=K$, we obtain a parametric curve on the Poincaré sphere:

$$
\left\{\begin{array}{l}
S_{x}= \pm \sqrt{S_{0}^{2}-K^{2}-\left(S_{0}^{2}-K^{2}\right)^{2}} \\
S_{y}=K^{2}-S_{0}^{2} \\
S_{z}=K
\end{array} .\right.
$$

These equations draw an eight-shaped line on the surface of the Poincaré sphere, as reported in Fig. 15. Similar calculations can be done in the general case with an arbitrary choice of the parameters $\left(J_{x}, J_{y}, J_{z}\right)$, and one still obtains an eightshaped line.

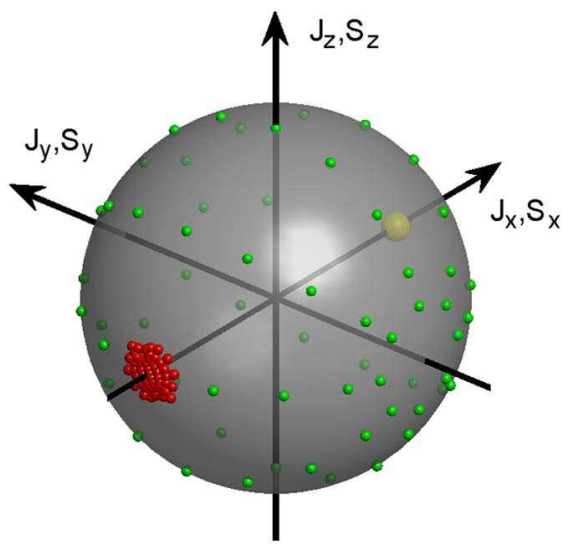

Fig. 12. (Color online) RBF model: Numerical simulations of the spatiotemporal system on the Poincaré sphere with $\Delta=0.05$. The green and red dots denote respectively the initial $(\vec{S}(0))$ and final $(\vec{S}(L))$ SOPs of the signal. The yellow dot displays the fixed pump SOP: $\vec{J}(L)=(1,0,0)$ for $L=15$.
It is important to note that there are two different restrictions to this phenomenon of attraction. The first one is due to the limit $J_{z}(L)= \pm J_{0}$, where Eq. (7) does not depend on $S_{x}(L)$ and $S_{y}(L)$. In this limit, Eq. (ㄱ) and Eq. (17) are not compatible, which means that the system no longer can relax toward the singular line of bitori.

This aspect is illustrated in Fig. 13, where we have computed the domain of existence of the attraction process in terms of $K$ in the special case $J_{x}(L)=0$. In this particular example, the equation of the border of the domain can be derived analytically. We first obtain $S_{y}$ as a function of $J_{z}$ and $K$ with the same method used for Eq. (20). Then the limit values $S_{y}= \pm 1$ lead to the following equation:

$$
K_{\lim }=\frac{3}{2} J_{z} \pm \sqrt{4-3 J_{z}^{2} \pm 4 \sqrt{1-J_{z}^{2}}} .
$$

It is clear in Fig. 13 that the domain of $K$ gets smaller as $J_{z}$ approaches its limit values.

The second restriction is the fact that the attraction process of the signal beam only takes place over half of the eightshaped line on the Poincaré sphere, a feature that has been established by the numerical simulations of the spatiotemporal Eqs. (1). This fact can also be easily explained by the nature of the singular bitori associated to the line of polarization attraction. Owing to the peculiar topology inherent to bitori, the stationary solutions can freely rotate around the bitorus, in contrast with trajectories that evolve on an ordinary pinched singular torus. This property allows for the existence of nonmonotonic stationary solutions, i.e., stationary solutions that exhibit an oscillatory behavior. This is illustrated in Fig. 14, which reports several stationary solutions chosen among the stationary solutions that lie on the closed singular curve on the Poincare sphere. Figure 14 (top) reports the stable stationary solutions, i.e., those that play the role of attractors for the spatiotemporal dynamics, whereas Fig. 14 (bottom) reports the unstable stationary solutions. Only the monotonic stationary solutions are stable, while the oscillatory ones are unstable. This feature corroborates the general observation pointed out originally in Refs. [4, $\underline{5}$ ], that only monotonic stationary solutions are attractors for the spatiotemporal dynamics.

Finally, another interesting consequence of the existence of this singular line of polarization attraction is the possibility to

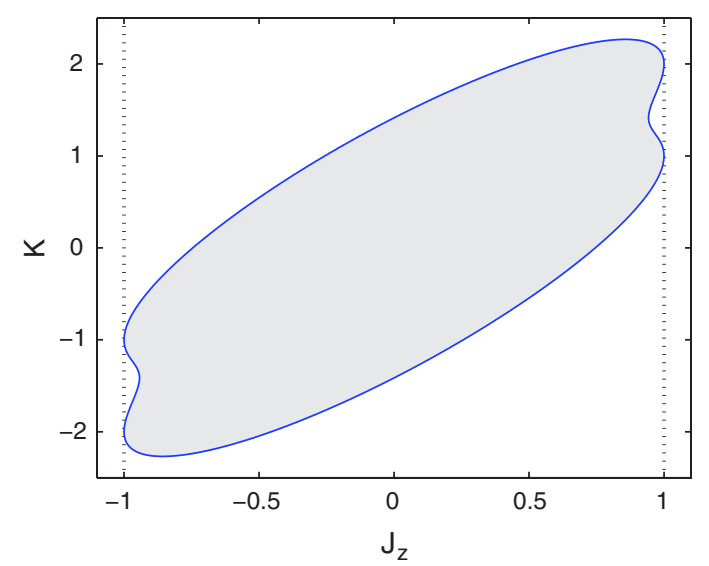

Fig. 13. (Color online) HBSF model: The gray domain depicts the possible values of $K$ as a function of $J_{z}(L)$ when $J_{x}(L)=0$. 

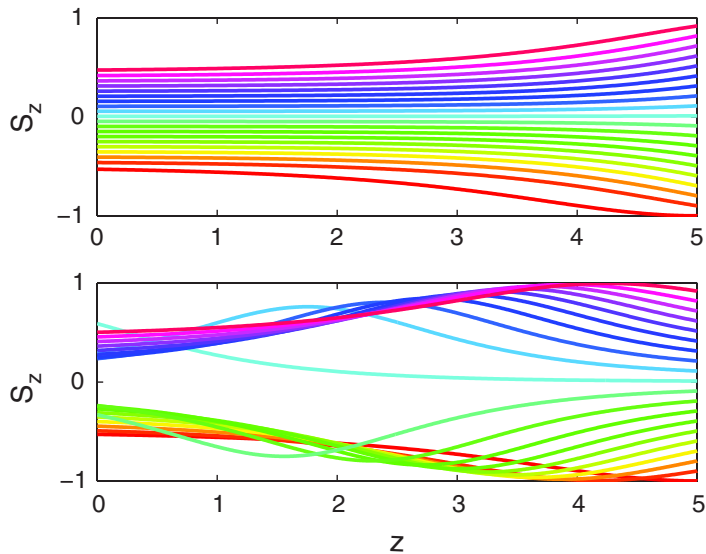

Fig. 14. (Color online) HBSF model: Monotonic (up) and nonmonotonic (bottom) behaviors of the stationary solutions corresponding respectively to the stable and unstable parts of the eight-shaped line. Only the monotonic stationary solutions are stable, whereas the stationary solutions that exhibit an oscillatory behavior are unstable. This explains why only half of the eight-shaped closed curve plays the role of an attractor in the spatiotemporal dynamics.

produce an elliptic polarization state from two linearly polarized states. Indeed, if both the signal and the pump beams are injected in a purely linear SOP in an $\mathrm{HBSF}$ with $\varphi=\pi / 4$, then the signal beam will be attracted toward a particular point on

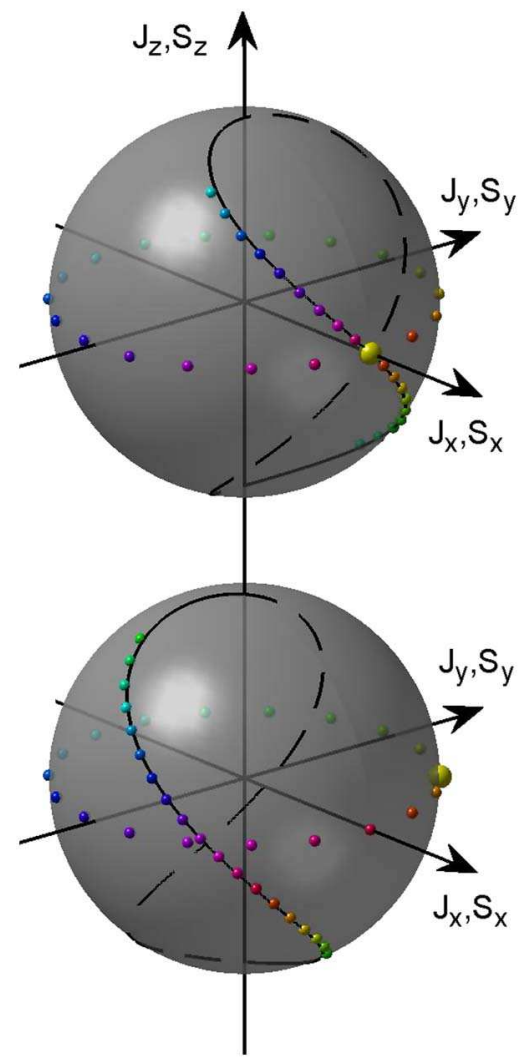

Fig. 15. (Color online) HBSF model: Two series of numerical simulations with two different values of $\vec{J}(L)$ that show the possibility of producing an elliptic polarization with two linear polarizations. The plain and dashed black lines depict respectively the stable and unstable parts of the figure eight. The two large yellow dots are associated to the two values of the pump $\vec{J}(L)$. The small dots on the equator depict the injected polarization signal $\vec{S}(0)$ and the small dots outside the equator (on eight-shaped curves) the outgoing polarization of the signal after the interaction. the eight-shaped closed curve at $z=L$, and its ellipticity will depend on the two angles that determine the initial linear SOPs of the pump and signal beams. A numerical study reveals that the angle that characterizes the linear SOP of the signal $\vec{S}(0)$ controls the position $\vec{S}(L)$ on the eight-shaped curve, while the angle of $\vec{J}(L)$ rotates the eight-curve around the $z$ axis. This is illustrated in Fig. 15, where the color code links the linear polarizations of $\vec{S}(0)$ to the corresponding elliptic polarizations of $\vec{S}(L)$ for a given linear polarization of $\vec{J}(L)$.

\section{CONCLUSION}

In summary, we have presented the mathematical techniques recently developed in order to provide a geometric approach for the study of Hamiltonian singularities of integrable systems. This theory sheds new light to the interpretation of the phenomenon of polarization attraction that occurs in counterpropagating optical beams in different types of optical fibers. We exposed in simple terms the theory of singular reduction, which permits to construct the energy-momentum diagram and characterizes the geometric properties of the underlying tori of the stationary system. We showed that the energy-momentum diagram allows us to determine the essential properties that characterize the process of polarization attraction. A brief overview of the different steps of this approach can be summarized as follows:

- Study of the stationary system (Hamiltonian, constant of the motion)

- Introduction of the invariant polynomials associated to the constant of the motion

- Analysis of the intersection of the energy surface with the reduced phase space

- Construction of the energy-momentum diagram and determination of the nature and of the position of the Hamiltonian singularities

We apply these tools in three different types of optical fibers. In the example of IFs, the energy-momentum diagram is characterized by one or two singularities, which leads to an efficient process of polarization attraction toward a defined set of SOPs. In RBFs, the singularities lie on the boundary of the energy-momentum diagram, which significantly reduces the efficiency of the attraction process. Finally, in HBSFs the energy-momentum diagram is characterized by the presence of a continuous line of singularities, which leads to a polarization attraction toward a continuous line of polarization states on the Poincaré sphere. We have completed previous results concerning the polarization attraction process in RBFs and HBSFs. The theory exposed here thus provides a rather complete understanding of the properties of the phenomenon of polarization attraction in these fiber systems. Note that, under rather general conditions, for both HBSFs and RBFs the efficiency of the attraction process increases as the fiber length and the powers of the beams increase. We also underline that all the analysis can be extended to unequal signal-pump powers: For HBSFs polarization attraction still occurs along a line of polarization states on the Poincare sphere, while for RBFs the singular torus is shown to split into two distinct singular tori for $S_{0} \neq J_{0}$, whose SOP coordinates read $S_{x}= \pm \rho J_{x}$, $S_{y}= \pm \rho J_{y}, S_{z}=-\rho J_{z}$, with $\rho=S_{0} / J_{0}$. For $\rho \simeq 1$ (within $10 \%)$, the simulations reveal an attraction toward the two 
SOPs states, while for higher values of $\rho$ the system exhibits a complex dynamics, including periodic behaviors that will be the subject of future investigations. We also point out that the rigorous mathematical proof of all these phenomena still constitutes an unsolved problem. A first step in this direction has been done in [35], where the relaxation process to a stationary state has been proven rigorously in the purely linear regime of the counterpropagating wave dynamics in a Bragg grating. Work is in progress in order to generalize these results to the nonlinear regime of the counterpropagating interaction. Besides the characterization of the phenomenon of polarization attraction, the theory exposed here can also be extended to study the stability properties of soliton solutions in a medium of finite extension. In a recent work it was shown that soliton solutions can become unstable due to the finite extension of the medium (e.g., a finite optical fiber length) [8]. In this way, the spatiotemporal dynamics relaxes toward a Hamiltonian singular state whose nature is completely different than that of the soliton state. More precisely, we showed that when the singular torus of the Hamiltonian system is isolated (as in IFs), then the space-time dynamics is asymptotically attracted toward the soliton solution, which is thus stable. Conversely, in HBSFs the soliton becomes unstable because of the presence of a continuous family of Hamiltonian singular tori and the space-time dynamics relaxes toward another stationary state of this family [10]. Work is in progress in order to extend this preliminary work to more general soliton systems, such as gap-solitons and three-wave interaction solitons.

\section{APPENDIX A: DERIVATION OF THE SOLITON SOLUTION}

In this appendix, we derive explicitly the form of the soliton solution for IF by assuming that this kind of solution lies on a singular torus [4,10]. This completes our presentation of these new Hamiltonian tools by showing their efficiency in the computation of soliton solutions. Similar computations could be done for HSBF and RBF. We start from Eq. (18) and we introduce a new pair of canonically conjugate coordinates $(J, \psi)$ and $(K, \chi)$, which are related to the old coordinates by the generating function $F=\left(I_{p}+I_{s}\right) \chi+I_{p} \psi$, such that

$$
\begin{aligned}
& \phi_{s}=\frac{\partial F}{\partial I_{s}}=\chi \\
& \phi_{p}=\frac{\partial F}{\partial I_{p}}=\chi+\psi \\
& K=\frac{\partial F}{\partial \chi}=I_{s}+I_{p} \\
& J=\frac{\partial F}{\partial \psi}=I_{p} .
\end{aligned}
$$

The Hamiltonian in these new coordinates can be written as

$$
H=2 \sqrt{\left(J_{0}^{2}-J^{2}\right)\left(S_{0}^{2}-(K-J)^{2}\right)} \cos \psi-\frac{1}{2}\left(J^{2}+(K-J)^{2}\right),
$$

which leads to the following dynamics:

$$
\frac{d J}{d \xi}=-\frac{\partial H}{\partial \psi}=2 \sqrt{\left(S_{0}^{2}-J^{2}\right)\left(J_{0}^{2}-(K-J)^{2}\right)} \sin \psi
$$

which can be expressed as

$$
\left(\frac{d J}{d \xi}\right)^{2}=e(J-\alpha)(J-\beta)(J-\gamma)(J-\delta),
$$

where $e>0$ and $\beta \leq \gamma \leq \delta \leq \alpha$, and the variable $\xi$ plays here the role of time. The solution of this equation can be written in terms of a Jacobi elliptic function [36]:

$$
J(\xi)=\beta+\frac{\gamma-\beta}{1-\eta \mathrm{sn}^{2}(\lambda \xi \mid \mu)},
$$

where $\eta=\frac{\gamma-\delta}{\beta-\delta}>0, \mu=\eta \frac{\beta-\alpha}{\gamma-\alpha}>0$, and $\lambda=\frac{1}{2} \sqrt{e(\gamma-\alpha)(\beta-\delta)}$ [37].

We recall that on a singular pinched torus, one of the fundamental periods is infinite, which entails $\mu=1$ since $\omega=\pi \lambda / Q P(\mu)$, where $Q P$ is the quarter-period of the Jacobi elliptic function. We thus set $\varepsilon=1-\mu$. We first have to compute $J(\xi+L / 4)$, where $L=2 \pi / \omega$. Here, we consider that the length of the fiber corresponds to one period of the solution. Then, a first-order Taylor series expansion in $\varepsilon$ gives

$$
J(\xi)=\frac{\delta(\beta-\gamma)-\beta(\delta-\gamma) e^{-2 \lambda \xi} \varepsilon^{1 / 2}}{\beta-\gamma-(\delta-\gamma) e^{-2 \lambda \xi} \varepsilon^{1 / 2}}+o(\varepsilon) .
$$

As $\varepsilon$ is a function of the four roots $\alpha, \beta, \gamma$, and $\delta$, this expression of $J$ only depends on these roots and on the fact that the system is close to the singular torus. One should also notice that this formula corresponds to a choice of specific boundary conditions and the whole family of soliton solutions can be obtained in the same way from Eq. (A1). The four roots have the form

$$
\begin{aligned}
\frac{K}{2} & \pm \frac{1}{6}\left(15 K^{2}+12 H \pm 12\left(K^{4}+16 S_{0}^{2} K^{2}\right.\right. \\
& \left.\left.+4 H K^{2}+4 S_{0}^{4}+8 S_{0}^{2} H+4 H^{2}\right)^{1 / 2}+48 S_{0}^{2}\right)^{1 / 2} .
\end{aligned}
$$

The next step of the computation consists in introducing the circular symmetry around the singular torus to write:

$$
\begin{gathered}
K=\rho \cos \theta \\
H=\rho \sin \theta-S_{0}^{2},
\end{gathered}
$$

where $\rho$ is a small parameter and $\theta \in[0,2 \pi]$. Performing a Taylor expansion of $J(\xi)$ in $\rho$ finally gives

$$
J(\xi)=S_{0} \tanh (\lambda \xi)+O(\rho) .
$$

Moreover, the first-order expansion of $L=2 \pi / \omega$ has the form

$$
\begin{aligned}
L= & \frac{2}{\sqrt{4-k^{2} S_{o}}}\left(2 \ln 2+\ln S_{0}^{2}\right. \\
& \left.-\ln \sqrt{-\frac{2 \cos ^{2} \theta-2+2 S_{0}^{2} k^{2} \cos ^{2} \theta-8 S_{0}^{2} \cos ^{2} \theta}{(k-2)^{2}(k+2)^{2}}}-\ln \rho\right) \\
& +O(\rho),
\end{aligned}
$$


which has a leading term in $\ln \rho$ for $\rho \ll 1$. This result allows us to characterize the polarization attraction in IF [5]. Because of the attracting properties of the singular torus, the spatiotemporal dynamics relax toward a stationary state that converges exponentially to the singular torus when the length $L$ of the fiber goes to infinity.

Returning back to the Cartesian coordinates, we need to determine $\psi$ and $\chi$. Using the coordinates $\left(H=-S_{0}^{2}, K=0\right)$ of the singular torus and the Hamiltonian

$$
H=2\left(S_{0}^{2}-J^{2}\right) \cos \psi-J^{2},
$$

we get that $\psi= \pm \frac{2 \pi}{3}$. The equation of motion on the singular torus $K=0$ implies $\chi=\chi(0)$, which gives

$$
\begin{aligned}
& S_{x}=S_{0} \cos (\chi(0)) \operatorname{sech}\left(\sqrt{3} S_{0} \xi\right) \\
& S_{y}=S_{0} \tanh \left(2 S_{0} \xi\right) \\
& S_{z}=S_{0} \sin (\chi(0)) \operatorname{sech}\left(\sqrt{3} S_{0} \xi\right) \\
& J_{x}=S_{0} \cos \left(\chi(0)+s \frac{2 \pi}{3}\right) \operatorname{sech}\left(\sqrt{3} S_{0} \xi\right) \\
& J_{y}=S_{0} \tanh \left(\sqrt{3} S_{0} \xi\right) \\
& J_{z}=S_{0} \sin \left(\chi(0)+s \frac{2 \pi}{3}\right) \operatorname{sech}\left(\sqrt{3} S_{0} \xi\right),
\end{aligned}
$$

with $s= \pm 1$. All these solutions are not symmetric with respect to $\xi$. The numerical simulations reveal that the spatiotemporal dynamics will select the symmetric solution. This leads us to choose the corresponding value of $\chi(0)$, i.e., $\chi(0)=$ $\frac{\pi}{6}$ and $s=1$. We thus recover the stable soliton solution considered in the numerical simulations in Ref. [10]:

$$
\begin{aligned}
& S_{x}=S_{0} \frac{\sqrt{3}}{2} \operatorname{sech}\left(\sqrt{3} S_{0} \xi\right) \\
& S_{y}=S_{0} \tanh \left(\sqrt{3} S_{0} \xi\right) \\
& S_{z}=S_{0} \frac{1}{2} \operatorname{sech}\left(\sqrt{3} S_{0} \xi\right) \\
& J_{z}=-S_{0} \frac{\sqrt{3}}{2} \operatorname{sech}\left(\sqrt{3} S_{0} \xi\right) \\
& J_{y}=S_{0} \tanh \left(\sqrt{3} S_{0} \xi\right) \\
& J_{z}=S_{0} \frac{1}{2} \operatorname{sech}\left(\sqrt{3} S_{0} \xi\right)
\end{aligned}
$$

\section{REFERENCES}

1. J. E. Heebner, R. S. Bennink, R. W. Boyd, and R. A. Fisher, "Conversion of unpolarized light to polarized light with greater than $50 \%$ efficiency by photorefractive two-beam coupling," Opt. Lett. 25, 257-259 (2000).

2. A. Picozzi, "Spontaneous polarization induced by natural thermalization of incoherent light," Opt. Express 16, 17171-17185 (2008).

3. S. Pitois, A. Picozzi, G. Millot, H. R. Jauslin, and M. Haelterman, "Polarization and modal attractors in conservative counterpropagating four-wave interaction," Europhys. Lett. 70, 88-94 (2005).

4. D. Sugny, A. Picozzi, S. Lagrange, and H. R. Jauslin, "Role of singular tori in the dynamics of spatiotemporal nonlinear wave systems," Phys. Rev. Lett. 103, 034102 (2009).
5. S. Lagrange, D. Sugny, A. Picozzi, and H. R. Jauslin, "Singular tori as attractors of four-wave-interaction systems," Phys. Rev. E 81, 016202 (2010).

6. E. Assemat, S. Lagrange, A. Picozzi, H. R. Jauslin, and D. Sugny, "Complete nonlinear polarization control in an optical fiber system,” Opt. Lett. 35, 2025-2027 (2010).

7. V. V. Kozlov and S. Wabnitz, "Theoretical study of polarization attraction in high birefringence and spun fibers," Opt. Lett. 35, 3949-3951 (2010).

8. V. V. Kozlov and S. Wabnitz, "Instability of optical solitons in the boundary value problem for a medium of finite extension," Lett. Math. Phys. 96, 405-413 (2011).

9. V. V. Kozlov, J. Nuno, and S. Wabnitz, "Theory of lossless polarization attraction in telecommunication fibers," J. Opt. Soc. Am. B 28, 100-108 (2011).

10. E. Assemat, A. Picozzi, H. R. Jauslin, and D. Sugny, "Instabilities of optical solitons and Hamiltonian singular solutions in a medium of finite extension," Phys. Rev. A 84, 013809 (2011).

11. E. Assémat, D. Dargent, A. Picozzi, H. R. Jauslin, and D. Sugny "Polarization control in spun and telecommunication optica fibers," Opt. Lett. 36, 4038-4041 (2011).

12. E. Assémat, C. Michel, A. Picozzi, H. R. Jauslin, and D. Sugny, "Manifestation of Hamiltonian monodromy in nonlinear wave systems," Phys. Rev. Lett. 106, 014101 (2011).

13. S. Pitois, J. Fatome, and G. Millot, "Polarization attraction using counterpropagating waves in optical fiber at telecommunication wavelengths," Opt. Express 16, 6646-6651 (2008).

14. J. Fatome, S. Pitois, P. Morin, and G. Millot, "Observation of light-by-light polarization control and stabilization in optical fibre for telecommunication applications," Opt. Express 18, 15311-15317 (2010).

15. V. V. Kozlov, J. Fatome, P. Morin, S. Pitois, G. Millot, and S. Wabnitz, "Nonlinear repolarization dynamics in optical fibers: transient polarization attraction," J. Opt. Soc. Am. B 28, 1782-1791 (2011).

16. P. Morin, J. Fatome, C. Finot, S. Pitois, R. Claveau, and G. Millot, "All-optical nonlinear processing of both polarization state and intensity profile for $40 \mathrm{Gbit} / \mathrm{s}$ regeneration applications," Opt. Express 19, 17158-17166 (2011).

17. D. J. Gauthier, M. S. Malcuit, A. L. Gaeta, and R. W. Boyd, "Polarization bistability of counterpropagating laser beams," Phys. Rev. Lett. 64, 1721-1724 (1990).

18. G. Gregori and S. Wabnitz, "New exact solutions and bifurcations in the spatial distribution of polarization in third-order nonlinear optical interactions," Phys. Rev. Lett. 56, 600-603 (1986).

19. A. L. Gaeta, R. W. Boyd, J. R. Ackerhalt, and P. W. Milonni, "Instabilities and chaos in the polarizations of counterpropagating light fields," Phys. Rev. Lett. 58, 2432-2435 (1987).

20. M. V. Tratnik and J. E. Sipe, "Nonlinear polarization dynamics. III. Spatial polarization chaos in counterpropagating beams," Phys. Rev. A 36, 4817-4822 (1987).

21. S. Trillo and S. Wabnitz, "Intermittent spatial chaos in the polarization of counterpropagating beams in a birefringent optical fiber," Phys. Rev. A 36, 3881-3884 (1987).

22. M. V. Tratnik and J. E. Sipe, "Nonlinear polarization dynamics. II. Counterpropagating-beam equations: New simple solutions and the possibilities for chaos," Phys. Rev. A 35, 2976-2988 (1987).

23. A. L. Gaeta and R. W. Boyd, "Transverse instabilities in the polarizations and intensities of counterpropagating light waves," Phys. Rev. A 48, 1610-1624 (1993).

24. S. Pitois, G. Millot, and S. Wabnitz, "Polarization domain wall solitons with counterpropagating laser beams," Phys. Rev. Lett. 81, 1409-1412 (1998).

25. S. Pitois, G. Millot, and S. Wabnitz, "Nonlinear polarization dynamics of counterpropagating waves in an isotropic optical fiber: theory and experiments," J. Opt. Soc. Am. B 18, 432-443 (2001).

26. S. Wabnitz, "Chiral polarization solitons in elliptically birefringent spun optical fibers," Opt. Lett. 34, 908-910 (2009).

27. D. David, D. D. Holm, and M. V. Tratnik, "Hamiltonian chaos in nonlinear optical polarization dynamics," Phys. Rep. 187, 281-367 (1990).

28. R. H. Cushman and L. M. Bates, Global Aspects of Classical Integrable Systems (Birkhauser, 1997). 
29. K. Efstathiou and D. A. Sadovskii, "Normalization and global analysis of perturbations of the hydrogen atom," Rev. Mod. Phys. 82, 2099-2154 (2010).

30. Y. S. Kivshar and G. P. Agrawal, Optical Solitons: From Fibers to Photonic Crystals (Academic Press, 2003).

31. E. Assémat, K. Efstathiou, M. Joyeux, and D. Sugny, "Fractional bidromy in the vibrational spectrum of HOCl," Phys. Rev. Lett. 104, 113002 (2010).

32. R. H. Cushman, H. R. Dullin, A. Giacobbe, D. D. Holm, M. Joyeux, P. Lynch, D. A. Sadovskii, and B. I. Zhilinskii, " $\mathrm{CO}^{2}$ molecule as a quantum realization of the $1: 1: 2$ resonant swingspring with monodromy," Phys. Rev. Lett. 93, 024302 (2004).
33. D. Sugny, P. Mardesic, M. Pelletier, J. Jebrane, and H. R. Jauslin, "Fractional Hamiltonian monodromy from a Gauss-Manin monodromy," J. Math. Phys. 49, 042701 (2008).

34. V. I. Arnold, Mathematical Methods of Classical Mechanics (Springer-Verlag, 1989).

35. M. Grenier, H.-R. Jauslin, C. Klein, and V. B. Matveev, "Wave attraction in resonant counter-propagating wave systems," J. Math. Phys. 52, 082704 (2011).

36. M. Abramowitz and I. A. Stegun, Handbook of Mathematical Functions (Dover Publications, 1965).

37. M. Joyeux, "Classical dynamics of the $1: 1,1: 2$ and 1:3 resonance Hamiltonians," Chem. Phys. 203, 281-307 (1996). 